\title{
Therapeutic Follicle Stimulating Hormone
}

National Cancer Institute

\section{Source}

National Cancer Institute. Therapeutic Follicle Stimulating Hormone. NCI Thesaurus.

Code C1822.

A therapeutic glycoprotein hormone synthesized by recombinant DNA technology or purified from biological sources, whose endogenous counterpart follicle stimulating hormone (FSH) stimulates and maintains the normal functions of reproductive system in males and females. ( $\mathrm{NCl} 04)$ 